\title{
A global multispecies single-fluid MHD study of the plasma interaction around Venus
}

\author{
Y. J. Ma, ${ }^{1}$ A. F. Nagy, ${ }^{2}$ C. T. Russell, ${ }^{1}$ R. J. Strangeway, ${ }^{1}$ H. Y. Wei, ${ }^{1}$ and G. Toth ${ }^{2}$ \\ Received 6 September 2012; revised 2 November 2012; accepted 25 November 2012; published 31 January 2013.
}

[1] This paper reports a new global multispecies single-fluid MHD model that was recently developed for Venus. This model is similar to the numerical model that has been successfully applied to Mars. Mass densities of proton and three important ionospheric ion species $\left(\mathrm{O}^{+}, \mathrm{O}_{2}^{+}\right.$, and $\left.\mathrm{CO}_{2}^{+}\right)$are self-consistently calculated in the model by including related chemical reactions and ion-neutral collision processes. The simulation domain covers the region from $100 \mathrm{~km}$ altitude above the surface up to $24 \mathrm{R}_{\mathrm{V}}$ in the tail. An adaptive spherical grid structure is constructed with radial resolution of about $5 \mathrm{~km}$ in the lower ionosphere. Bow shock locations are well reproduced for both solar-maximum and solar-minimum conditions using appropriate solar wind parameters for each case. It is shown that the shock locations are farther from the planet during the solar maximum condition, because of both the enhanced solar radiation strength and the relatively small Mach number. The simulation results also agree well with Venus Express observations, as shown by comparisons between model results with magnetic fields observed by the spacecraft.

Citation: Ma, Y. J., A. F. Nagy, C. T. Russell, R. J. Strangeway, H. Y. Wei, and G. Toth (2013), A global multispecies single-fluid MHD study of the plasma interaction around Venus, J. Geophys. Res. Space Physics, 118, 321-330, doi:10.1029/2012JA018265.

\section{Introduction}

[2] Magnetometer observation of Pioneer Venus Orbiter (PVO) confirmed the weakness of the intrinsic field of the planet (equatorial surface field $<0.5 \mathrm{nT}$ from an intrinsic dipole) [Phillips and Russell, 1987]. Without the shielding of a strong intrinsic magnetic field, the Venusian atmosphere and ionosphere interact directly with the solar wind plasma.

[3] Because of the supermagnetosonic nature of the solar wind and the high conductivity of the ionosphere, three important plasma boundaries typically form in the interaction region: bow shock, magnetic pile-up boundary (the induced magnetopause) and ionopause. The location of these plasma boundaries and the structure of the ionosphere are very much dependent on solar activity and the dynamic pressure of the solar wind [Russell et al., 2006]. The bow shock location during solar minimum conditions is $2.14 \mathrm{R}_{\mathrm{V}}$ near the terminator, $1600 \mathrm{~km}$ closer to Venus than the $2.40 \mathrm{R}_{\mathrm{V}}$ determined during solar maximum conditions [Zhang et al., 2008].

\footnotetext{
${ }^{1}$ Institute of Geophysics and Planetary Physics, UCLA, Los Angeles, California, USA.

${ }^{2}$ Department of Atmospheric, Oceanic and Space Sciences, University of Michigan, Ann Arbor, Michigan, USA.

Corresponding author: Y. J. Ma, Institute of Geophysics and Planetary Physics, UCLA, Los Angeles, California, 90025, USA. (yingjuan@igpp.ucla.edu)

(C)2012. American Geophysical Union. All Rights Reserved. 2169-9380/13/2012JA018265
}

[4] The solar wind interaction with Venus has been studied with many numerical models, including the gas dynamic models [Spreiter and Stahara, 1980; Stahara et al., 1987; Spreiter and Stahara, 1992], MHD models [McGary and Pontius, 1994; Cable and Steinolfson, 1995; Tanaka et al., 1997; Tanaka 2000; Kallio et al., 1998; Bauske et al., 1998; Terada et al., 2009] and hybrid models [Terada et al., 2002, 2004; Kallio et al., 2006, 2008].

[5] Gasdynamic models by Spreiter and Stahara [1980] assumed that the ionopause is the obstacle to the solar wind flow. The predicted shock location is closer to Venus than observed, which suggests that mass loading plays an important role in determining the shock location [Slavin et al., 1980]. Belotserkovskii et al. [1987] and Breus et al. [1987] presented results from mass loaded gasdynamic calculations, and they found that the mass loading moved the terminator shock outward to a sufficient extent to match the observations. While Spreiter and Stahara [1992], using their gasdynamic model concluded that mass loading has little effect on the bow shock positions. Zhang et al. [1991] suggested that the actual obstacle is larger than that resulting from the ionopause, namely that it is really the Venus ionosphere plus the magnetic barrier, which could not be reproduced by gas dynamic models [Russell et al., 2006].

[6] The single-fluid MHD model treats the ionosphere as a perfectly conducting sphere. Thus, it is able to reproduce the magnetic barrier above the obstacle. Early two-dimensional MHD models [McGary and Pontius, 1994; Murawski and Steinolfson, 1996] also found that the mass loading causes the bow shock to move outward from the planet, but not as 
far as observed at Venus during solar maximum. However, these models used only photoionization of hot oxygen, and assumed no dependence on the solar zenith angle (SZA). Kallio et al. [1998] included EUV ionization of hot and cold oxygen, and they multiplied the photoionzation rate by a factor of 3 to account for the electron impact ionization and charge exchange, in order to get shock locations comparable to the observation. Bauske et al. [1998] incorporated comprehensive mass loading (including SZA-dependent photoionization, impact ionization, and charge exchange) and also took into account the mass change of the plasma near the ionopause. They found that electron impact ionization and charge exchange are less significant for mass loading than photoionization. None of these early MHD models were able to incorporate a realistic ionosphere in the model, because of the small scales that are required to resolve the ionospheric structure. Tanaka and Murawski [1997] made the first attempt to include a self-consistent ionosphere by using a two-species $(\mathrm{O}+$ and $\mathrm{H}+$ ) single-fluid model (assuming the two ion species move at the same velocity). They used adaptive grids in spherical coordinates with very high spatial resolution: $15 \mathrm{~km}$ near the ionopause. Although their ionopause location was slightly higher than the observation and their model only allowed magnetic fields perpendicular to the flow direction, their model was the first global model that successfully reproduced the ionopause. A recent paper by Terada et al. [2009] uses a comprehensive three-dimensional MHD model with 10-ion- species to study the solar wind interaction with the ionosphere of Venus. This model successfully reproduced many observed features of the ionospheric structures and its dynamics. The model obtained reasonable but slightly high bow shock and ionopause locations with the selected parameters for solar maximum condition. They also found that inclusion of a thermal conductivity term leads to realistic plasma temperature profiles in the ionosphere.

[7] This paper presents results from a sophisticated fourspecies single-fluid global MHD model of Venus, adopted from the Mars model [Ma et al., 2004a]. This model has very high radial resolution $(5 \mathrm{~km})$ in the Venus ionosphere region. The next section discusses details of the Venus MHD model, including the equations solved by the model and the model inputs. Section 3 shows the numerical results obtained for solar maximum and solar minimum cases. Simulation results of a case study and comparison with observations are presented in section 4. Some discussion and summary are given in section 5 .

\section{Model Description}

[8] The MHD model for Venus is adapted from the global multispecies MHD models of Mars [Ma et al., 2004a; Ma and Nagy, 2007] and Titan [Ma et al., 2004b, 2006, 2007]. All these models use the BATS-R-US (Block Adaptive-Tree Solar-wind Roe-type Upwind Scheme) code of the University of Michigan [Powell et al., 1999; Tóth et al., 2012].

\subsection{MHD Equations}

[9] The following set of equations is used to describe the plasma interaction around Venus to self-consistently include the effect of the atmosphere and ionosphere:

\section{Continuity equations}

$$
\begin{aligned}
& \frac{\partial \rho_{i}}{\partial t}+\nabla \cdot\left(\rho_{i} \mathbf{u}\right)=S_{i}-L_{i} \\
& S_{i}=m_{i} N_{i}\left(v_{\mathrm{ph}, i}+v_{\mathrm{imp}, i}+\sum_{s=\text { ions }} k_{s i} n_{s}\right) \\
& L_{i}=m_{i} n_{i}\left(\alpha_{R, i} n_{e}+\sum_{t=\text { neutrals }} k_{i t} n_{t}\right)
\end{aligned}
$$

Momentum equation

$$
\begin{gathered}
\frac{\partial(\rho \mathbf{u})}{\partial t}+\nabla \cdot\left(\rho \mathbf{u u}+p \mathbf{I}+\frac{B^{2}}{2 \mu_{0}} \mathbf{I}-\frac{1}{\mu_{0}} \mathbf{B B}\right) \\
=\rho \mathbf{G}-\sum_{i=\text { ions }} \rho_{i} \sum_{t=\text { neutrals }} v_{i t} \mathbf{u}-\sum_{i=\text { ions }} L_{i} \mathbf{u}
\end{gathered}
$$

\section{Magnetic induction equation}

$$
\frac{\partial \mathbf{B}}{\partial t}+\nabla \cdot(\mathbf{u B}-\mathbf{B u})=-\nabla \times\left(\frac{1}{\mu_{0} \sigma_{0}} \nabla \times B\right)
$$

\section{Energy equation}

$$
\begin{aligned}
& \frac{\partial \varepsilon}{\partial t}+\nabla \cdot\left(\mathbf{u}\left[\varepsilon+p+\frac{1}{2 \mu_{0}} B^{2}\right]-\frac{1}{\mu_{0}}(\mathbf{B} \cdot \mathbf{u}) \mathbf{B}+B \times \frac{\nabla \times B}{\mu_{0} \sigma_{0}}\right) \\
& =\rho \mathbf{u} \cdot \mathbf{G}-\sum_{i=\text { ions }} \sum_{t=\text { neutrals }} \frac{\rho_{i} v_{i t}}{m_{i}+m_{t}}\left[3 k\left(T_{n}-T_{i}\right)-m_{i} u^{2}\right] \\
& -\frac{1}{2} \sum_{i=\text { ions }} L_{i} \mathbf{u}^{2}+\frac{k}{\gamma-1} \sum_{i=\text { ions }}\left(\frac{S_{i} T_{n}-L_{i} T_{i}}{m_{i}}-\frac{\rho_{i}}{m_{i}} \alpha_{R, i} n_{e} T_{e}\right)
\end{aligned}
$$

where $\rho_{i}(i=1$ to 4$)$ are the mass densities of $\mathrm{H}^{+}, \mathrm{O}_{2}^{+}, \mathrm{O}^{+}$, and $\mathrm{CO}_{2}^{+}$, respectively. $n_{i}$ are the corresponding number densities, and $S_{i}$, and $L_{i}$ are the corresponding mass production and loss rates. In the model, the Venus neutral atmosphere is represented by two species $\left(\mathrm{CO}_{2}\right.$ and $\left.\mathrm{O}\right)$, their densities are noted by $N_{i}$. The mass production and loss rates are calculated based on photo- and impact ionization (with rates $v_{\mathrm{ph}, i}$ and $\left.v_{\mathrm{imp}, i}\right)$ of each neutral, charge exchange $\left(k_{s i}\right)$ between the major neutral and ion species and recombination reactions (with rates $\alpha_{R, i}$ ). Some of the reaction rates are temperature-dependent as listed in Table 1 . In order to evaluate these rates, we assume that the electron and ion temperatures are approximately equal and given by $T_{\mathrm{p}} / 2$, half of the plasma temperature.

[10] The total mass density $\rho$ is defined as

$$
\rho=\sum_{i=\text { ions }} \rho_{i}
$$

and $\varepsilon$ is the total energy density, defined as

$$
\varepsilon=\frac{1}{2} \rho u^{2}+\frac{1}{\gamma-1} p+\frac{1}{2 \mu_{0}} B^{2}
$$

[11] Also in equations (3) and (4), $v_{i t}$ is the ion neutral collision frequency (approximated to be $4 \times 10^{-10}\left\{[\mathrm{O}]+\left[\mathrm{CO}_{2}\right]\right\} \mathrm{s}^{-1}$ ) [Schunk and Nagy,1980], $T_{\mathrm{n}}$ is the temperature of the newly produced ions, which is assumed to be $1000 \mathrm{~K}$ at solar 


\section{MA ET AL.: MHD STUDY OF PLASMA INTERACTION AROUND VENUS}

Table 1. List of Chemical Reactions and Rates Considered in the Model ${ }^{\mathrm{a}}$

\begin{tabular}{lll}
\hline Reaction & Rate Coefficient & References \\
\hline $\mathrm{CO}_{2}+\mathrm{h} v \rightarrow \mathrm{CO}_{2}^{+}+\mathrm{e}$ & $3.24 \times 10^{-6} \mathrm{~s}^{-1}$ (solar max) & Schunk and Nagy [2009] \\
$\mathrm{O}+\mathrm{h} v \rightarrow \mathrm{O}^{+}+\mathrm{e}$ & $1.28 \times 10^{-6} \mathrm{~s}^{-1}$ (solar min) & Schunk and Nagy [2009] \\
& $1.21 \times 10^{-6} \mathrm{~s}^{-1}$ (solar max) & \\
$\mathrm{CO}_{2}^{+}+\mathrm{O} \rightarrow \mathrm{O}_{2}^{+}+\mathrm{CO}$ & $4.67 \times 10^{-7} \mathrm{~s}^{-1}($ solar min) & Schunk and Nagy [2009] \\
$\mathrm{CO}_{2}^{+}+\mathrm{O} \rightarrow \mathrm{O}^{+}+\mathrm{CO} 2$ & $1.64 \times 10^{-10} \mathrm{~cm}^{-3} \mathrm{~s}^{-1}$ & Schunk and Nagy [2009] \\
$\mathrm{O}^{+}+\mathrm{CO}_{2} \rightarrow \mathrm{O}_{2}^{+}+\mathrm{CO}$ & $9.60 \times 10^{-11} \mathrm{~cm}^{-3} \mathrm{~s}^{-1}$ & Fox and Sung [2001] \\
& $1.1 \times 10^{-9} \mathrm{~cm}^{-3} \mathrm{~s}^{-1}$ for $T_{\mathrm{i}} \leq 800 \mathrm{~K} ;$ & \\
& $1.1 \times 10^{-9}\left(800 / T_{\mathrm{i}}\right)^{0.39} \mathrm{~cm}^{-3} \mathrm{~s}^{-1}$ & Schunk and Nagy [2009] \\
$\mathrm{H}^{+}+\mathrm{O} \rightarrow \mathrm{O}^{+}+\mathrm{H}$ & for $T_{\mathrm{i}}>800 \mathrm{~K}$ & Schunk and Nagy [2009] \\
$\mathrm{O}_{2}^{+}+\mathrm{e} \rightarrow \mathrm{O}+\mathrm{O}$ & $3.75 \times 10^{-10} \mathrm{~cm}^{-3} \mathrm{~s}^{-1}$ & \\
& $1.95 \times 10^{-7}\left(300 / T_{\mathrm{e}}\right)^{0.7} \mathrm{~cm}^{-3} \mathrm{~s}^{-1}$ & \\
& for $T_{\mathrm{e}} \leq 1200 \mathrm{~K} ;$ & Fox and Sung [2001] \\
\hline $\mathrm{CO}_{2}^{+}+\mathrm{e} \rightarrow \mathrm{CO}+\mathrm{O}$ & $7.38 \times 10^{-8}\left(1200 / \mathrm{T}_{\mathrm{e}}\right)^{0.56} \mathrm{~cm}^{-3} \mathrm{~s}^{-1}$ & for $T_{\mathrm{e}}>1200 \mathrm{~K} ;$ \\
\hline
\end{tabular}

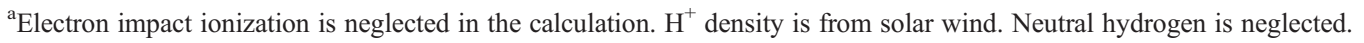

minimum and $1500 \mathrm{~K}$ at solar maximum. The ratio of specific heats $\gamma$ is taken to be $5 / 3$. The other symbols have their usual definitions.

[12] In equation (3), $\sigma_{0}$ is the electrical conductivity of the plasma. In the model the electrical conductivity is calculated using

$$
\sigma_{0}=\frac{n_{e} e^{2}}{m_{e}\left(v_{\mathrm{ei}}+v_{\mathrm{en}}\right)}
$$

where $m_{\mathrm{e}}$ is the electron mass, $v_{\mathrm{ei}}$ and $v_{\mathrm{en}}$ are the electron-ion and electron-neutral collision frequencies, respectively. The collision frequencies $v_{\mathrm{ei}}$, and $v_{\mathrm{en}}$ are taken from Schunk and Nagy [1980], where

$$
v_{\mathrm{ei}}=54.5 n_{i} / T_{e}^{3 / 2}
$$

and

$$
\begin{aligned}
v_{\mathrm{en}}= & 3.68 \times 10^{-8}\left(1+4.1 \times 10^{-11}\left|4500-T_{e}\right|^{2.93}\right)\left[\mathrm{CO}_{2}\right] \\
& +8.9 \times 10^{-11}\left(1+5.7 \times 10^{-4} T_{e}\right) T_{e}^{1 / 2}[\mathrm{O}]
\end{aligned}
$$

[13] The above set of equations is solved using an upwind finite-volume scheme based on an approximate Riemann solver. The simulation starts with a two-stage local time stepping scheme, which allows different grid cells to advance with different time steps, thus accelerating convergence toward a steady state. Because of the stiffness of the source terms, a point implicit scheme is used to evaluate them. After the results get to a quasi-steady state, a timedependent scheme is used for $1 \mathrm{~h}$ physical simulation time.

\subsection{Chemical Model and Model Inputs}

[14] We adopted a spherically symmetric neutral atmosphere as the background to provide various reaction rates. The neutral atmosphere is simplified to contain only two neutrals: $\mathrm{CO}_{2}$ and $\mathrm{O}$, in which the former one is typically the predominant species below around $160 \mathrm{~km}$ altitude, while the latter one becomes the main component above that altitude. The densities of the two neutrals are based on the atmospheric model of Fox and Sung [2001] for solar max and solar min conditions. For solar max,

$$
\begin{gathered}
{\left[\mathrm{CO}_{2}\right]=1.0 \times 10^{15} \times \mathrm{e}^{-(\mathrm{z}-\mathrm{z} 0) / 5.5} \mathrm{~cm}^{-3}} \\
{[\mathrm{O}]=2.0 \times 10^{11} \times \mathrm{e}^{-(\mathrm{z}-\mathrm{z} 0) / 17.0} \mathrm{~cm}^{-3}}
\end{gathered}
$$

[15] For solar min:

$$
\begin{gathered}
{\left[\mathrm{CO}_{2}\right]=1.0 \times 10^{15} \times \mathrm{e}^{-(\mathrm{z}-\mathrm{z} 0) / 5.1} \mathrm{~cm}^{-3}} \\
{[\mathrm{O}]=1.3 \times 10^{11} \times \mathrm{e}^{-(\mathrm{z}-\mathrm{z} 0) / 15.5} \mathrm{~cm}^{-3}}
\end{gathered}
$$

where $\mathrm{z}$ is altitude in kilometers and $\mathrm{z} 0=100 \mathrm{~km}$ is the altitude of the inner boundary of the model. The neutral densities for solar maximum and solar minimum conditions as used in the model are shown by the solid lines and dashed lines respectively in the left panel of Figure 1. We approximate the optical depth effect for the photoionization rates, by including a cosine factor for the different solar zenith angles and by assuming average absorption coefficients of $2.6 \times 10^{-17}$ and $1.5 \times 10^{-17} \mathrm{~cm}^{2}$, for $\mathrm{CO}_{2}$ and $\mathrm{O}$, respectively [Schunk and Nagy, 2009]. On the night side, the solar flux was assumed to be $1.0 \times 10^{-5}$ times the unattenuated solar radiation in order to avoid zeros. The production rates used in the model at $60^{\circ} \mathrm{SZA}$ are also shown in the right panel of Figure 1 for both solar cycle conditions. The neutral densities at low altitudes are similar, but drop faster in the case of solar minimum condition due to the smaller scale height. As a result, the peak of the production rates is slightly lower in the solar minimum case.

[16] The Venus Solar Orbital coordinates are used in the calculation. It is a Venus-centered system with the $X$ axis toward the Sun, the $Z$ axis northward and perpendicular to the orbital plane, and the $Y$ axis completing the right hand system. The computational domain is defined by $-24 R_{\mathrm{V}} \leq X \leq$ $8 R_{\mathrm{V}},-16 R_{\mathrm{V}} \leq Y, Z \leq 16 R_{\mathrm{V}}$, where $R_{\mathrm{V}}=6052 \mathrm{~km}$ is the radius of Venus. The inner boundary was taken to be $100 \mathrm{~km}$ above the Venus surface. A spherical grid structure is used in the calculation. The angular resolution is kept to be $2.5^{\circ}$ both azimuthally and longitudinally in the computation domain. The radial resolution is $5 \mathrm{~km}\left(\sim 0.0008 R_{\mathrm{V}}\right)$ from the lower 

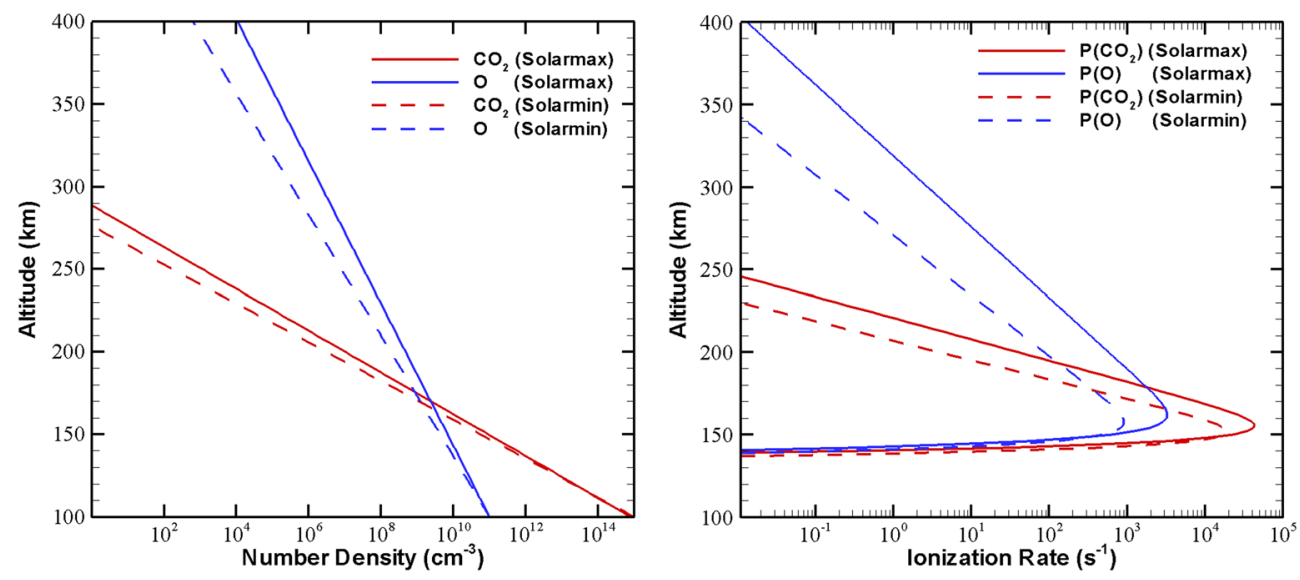

Figure 1. Neutral profiles and photoionzation production rates (at $60 \mathrm{SZA}$ ) used in the simulation (based on the atmospheric model of Fox and Sung [2001].

boundary $(100 \mathrm{~km})$ to $500 \mathrm{~km}$ altitude, above this altitude, the radial resolution gradually increased from $5 \mathrm{~km}$ to $2000 \mathrm{~km}$ $\left(0.34 R_{\mathrm{V}}\right)$ near the outer boundary at $24 R_{\mathrm{V}}$ downstream. Such a grid contains $480 \times 144 \times 72=5.0$ million cells in total. The simulation was performed on the NASA Columbia (PLEIADES) computer, with about 12,000 cpu hour/job.

[17] The $\mathrm{O}_{2}^{+}, \mathrm{O}^{+}$, and $\mathrm{CO}_{2}^{+}$densities at the inner boundary were taken to be the photochemical equilibrium values. An absorbing boundary has been used for $\mathbf{u}$ and $\mathbf{B}$; which enforces zero gradients of both vectors. This boundary condition allows the magnetic field to diffuse into the inner boundary. At about $100 \mathrm{~km}$, chemistry is dominant, and strong ion-neutral collisions enforce steady plasma. The strong magnetic diffusion coefficient enforces nearly zero gradient of the magnetic field. The plasma temperature (sum of the electron and ion temperatures) at the inner boundary was assumed to be two times the corresponding neutral temperature $(200 \mathrm{~K})$, and the pressure was set accordingly.

\section{Simulation Results for Solar Maximum and Solar Minimum Cases}

[18] Two different cases have been simulated for solar maximum and solar minimum condition of Venus, using the multispecies single-fluid MHD model with magnetic resistivity included. The solar wind parameters were chosen based on Luhmann et al. [1993]. The specific values are listed in Table 2. For both cases, the plasma flow is assumed to be along the $X$ direction and the magnetic field is assumed to be a Parker spiral in the $X-Y$ plane with $36^{\circ}$ spiral angle. The set of parameters corresponds to a solar wind dynamic pressure of $4.5 \mathrm{nPa}$ for solar maximum case and $6.4 \mathrm{nPa}$ for solar minimum case. They are consistent with solar wind conditions at $0.72 \mathrm{AU}$ based on PVO observations [Russell et al., 2006]. The plasma beta is 0.66 and the fast magnetosonic Mach number is 4.2 for solar maximum case, while the corresponding numbers are 2.5 and 5.6 for the solar minimum case.

[19] Figure 2 shows the contour of magnetic field and plasma flow speed in meridian $(X Z)$ plane and equatorial $(X Y)$ planes for solar maximum case. The color plots show the magnitudes; the white arrows show the direction of the magnetic field (left panels) and plasma flow (right panels) projected in the corresponding planes. The black dashed line is the average bow shock location at solar maximum condition from Zhang et al. [1990], taken to be $R=2.40$ / $\left(1+0.66^{*} \cos (\mathrm{SZA})\right)$.

[20] The solar wind plasma flow is significantly slowed down across the shock and diverted around the obstacle. The flow speed close to the body is nearly stagnated due to intensive mass loading and collisions with neutral atmosphere. The flow pattern in the plasma wake region is rather complicated. Part of the flow is coming back to the planet to maintain the nightside ionosphere, part of the flow is traveling away from the planet to contribute to the escape of the plasma. The magnetic field increases across the shock and wraps around the obstacle in the $X Y$ plane. There is a significant pile-up of the field lines above the ionosphere. The magnetic field contour is quite different in the $X Z$ plane than the equatorial plane. The differences are caused by the direction of the magnetic field. In the equatorial plane, the plane contains the magnetic field lines, and the field lines wrap around the obstacle, so the magnetic field is rather strong close to the obstacle even in the tail region. In the $X Z$ plane, on the other hand, the magnetic field lines slip over the obstacle. Because the flow dynamic pressure only pushes the plasma along the flow direction, the field strength in the wake region is not as strong as in the $X Y$ plane. This is also seen in Figure 3, which shows both the upstream and downstream views of the interaction process. The gray isosurface represents a density contour with planetary $\mathrm{O}^{+}$density equals to $100 / \mathrm{cc}$ as an indication of the outer boundary of Venus ionosphere, which is around $400 \mathrm{~km}$ altitude along the subsolar line, but extends nearly to $3 R_{\mathrm{V}}$ in the nightside. This figure shows how field lines wrap around the obstacle on the upstream side, but slip over the obstacle on the night

Table 2. List of Solar Wind Parameters for Selected Cases

\begin{tabular}{llccc}
\hline & $\begin{array}{c}\text { Density } \\
\left(\mathrm{cm}^{-3}\right)\end{array}$ & $\begin{array}{c}\text { Velocity } \\
(\mathrm{km} / \mathrm{s})\end{array}$ & $\begin{array}{c}\text { Magnetic Field } \\
(\mathrm{nT})\end{array}$ & $\begin{array}{c}\text { EUV } \\
\text { Strength }\end{array}$ \\
\hline Case 1-Solarmax & 17 & $(-400,0,0)$ & $(12.1,-8.8,0.0)$ & Solar max \\
Case 2-Solarmin & 22 & $(-420,0,0)$ & $(7.8,-5.6,0.0)$ & Solar min \\
Case 3-VEX & 12 & $(-430,0,0)$ & $(5.0,1.0,-3.8)$ & Solar min \\
\hline
\end{tabular}



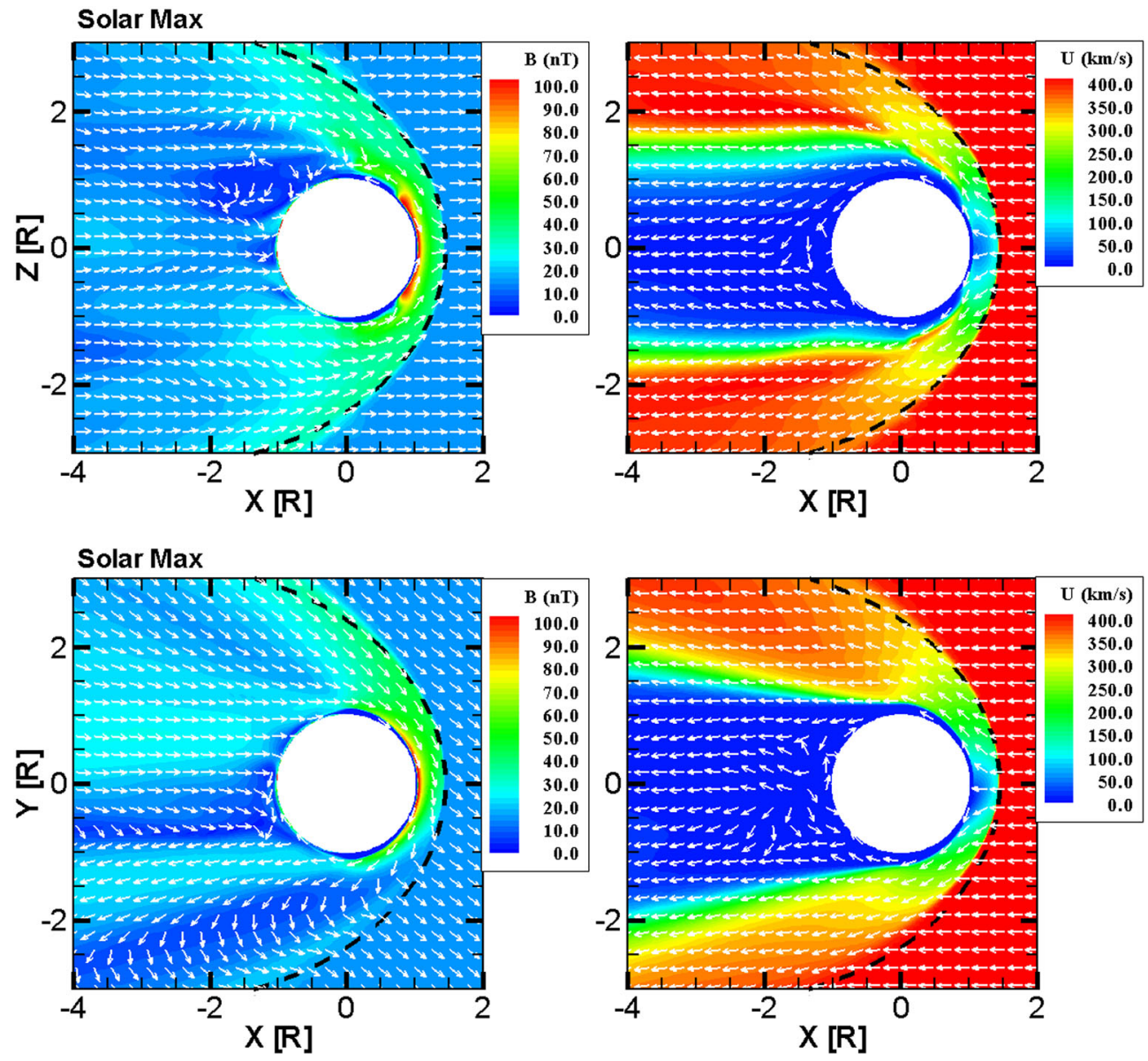

Figure 2. The contour of magnetic field and plasma flow speed in meridian $(X Z)$ plane and equatorial $(X Y)$ planes for solar maximum case. The color plots show the magnitudes; the white arrows show the direction of the magnetic field (left panels) and plasma flow (right panels) projected in the corresponding planes. The black dashed line is the average bow shock location at solar maximum condition from Zhang et al. [1990].
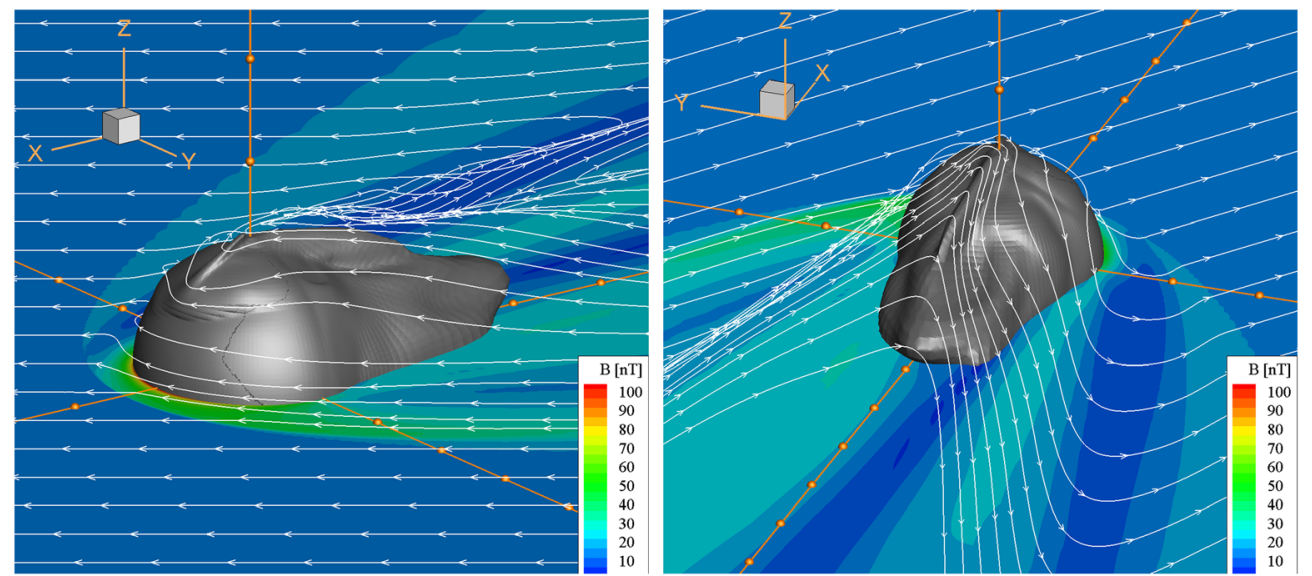

Figure 3. Three-dimensional plot of solar wind interaction with Venus for solar max case, with the left panel showing an upstream view, while the right panel a downstream view. The white lines with arrows are magnetic field lines. The gray isosurface represents a density contour with planetary $\mathrm{O}^{+}$density equals to $100 \mathrm{~cm}^{-3}$ as an indication of Venus ionopause. 

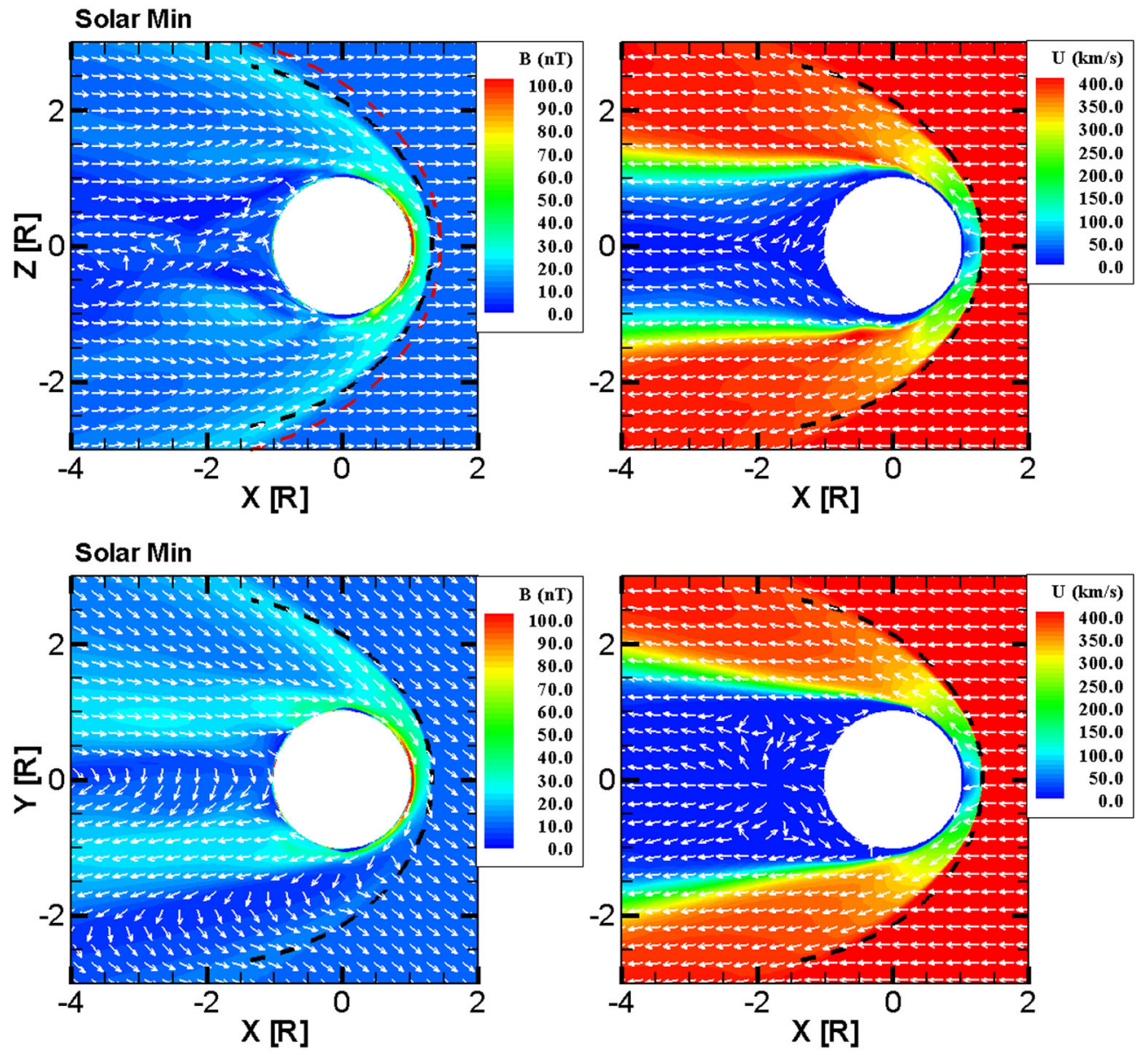

Figure 4. Same as Figure 2 but for solar minimum case. The black dashed line represents the mean bow shock locations at solar minimum condition from Zhang et al. [2008]. The average bow shock location for solar maximum condition from Zhang et al. [1990] is also plotted in red dashed line in the top left panel for comparison.

side. The field lines in the high latitude of the front side of the obstacle are rotated because of velocity shear across the composition boundary as suggested by Strangeway and Russell [1996].

[21] The interaction pattern is rather symmetric in the $X Z$ plane as shown in the upper panels of the Figure 2. The calculated bow shock location agrees well with the observations especially in the upstream side of the interaction region in the plane. There is a small discrepancy at the flank region when $X<0$. The simulation results also show a clear dawndusk asymmetry in the $X Y$ plane, with the quasi-parallel shock being closer to the planet than the quasi-perpendicular shock. Such a feature is consistent with PVO observations [Zhang et al., 1991]. There is a current sheet in the XY plane tilted toward the negative $Y$ direction.

[22] Figure 4 is a similar plot of Figure 2 but for the solar minimum case. The black dashed line represents the mean bow shock locations at solar minimum condition from Zhang et al. [2008], taken to be $R=2.14 /(1+0.62 * \cos$ (SZA)). The general interaction pattern is similar to the solar maximum condition except that the bow shock location is closer to the planet and the plasma wake region is narrower. This is due to a weaker ionospheric obstacle and a higher solar wind pressure for the solar minimum case.
[23] Based on PVO and Venus Express (VEX) observations, the average subsolar shock locations are $1.44 R_{\mathrm{V}}$ and $1.32 R_{\mathrm{V}}$ for solar maximum and solar minimum conditions respectively. The calculated subsolar shock location for the solar max case is $1.42 R_{\mathrm{V}}$, slightly smaller than the mean observational value. The modeled subsolar shock location for solar min case is $1.31 R_{\mathrm{V}}$, matching very well with the observations. The shock locations are farther from the planet during the solar maximum condition because of both the enhanced solar radiation strength and the relatively small Mach number. Simulation results also show that the dawn-dusk asymmetry is more apparent in the solar maximum case.

[24] We also ran cases with no mass loading (by turning off all the chemical reactions) for the same set of solar wind conditions, and the predicted subsolar shock location were $1.32 R_{\mathrm{V}}$ and $1.25 R_{\mathrm{V}}$ for solar max and solar min cases, respectively. The shock location moves $0.10 R_{\mathrm{V}}$ and $0.06 R_{\mathrm{V}}$ outward for solar max and solar min cases respectively due to the mass loading. Considering the fact that the grid resolution is around $40 \mathrm{~km}\left(0.007 R_{\mathrm{V}}\right)$ near the shock location, our results clearly demonstrated that the mass loading and is important in determining the shock location. The ion-neutral collision could also contribute to slow down the shocked solar wind and thus has some effect in determining the shock location. 

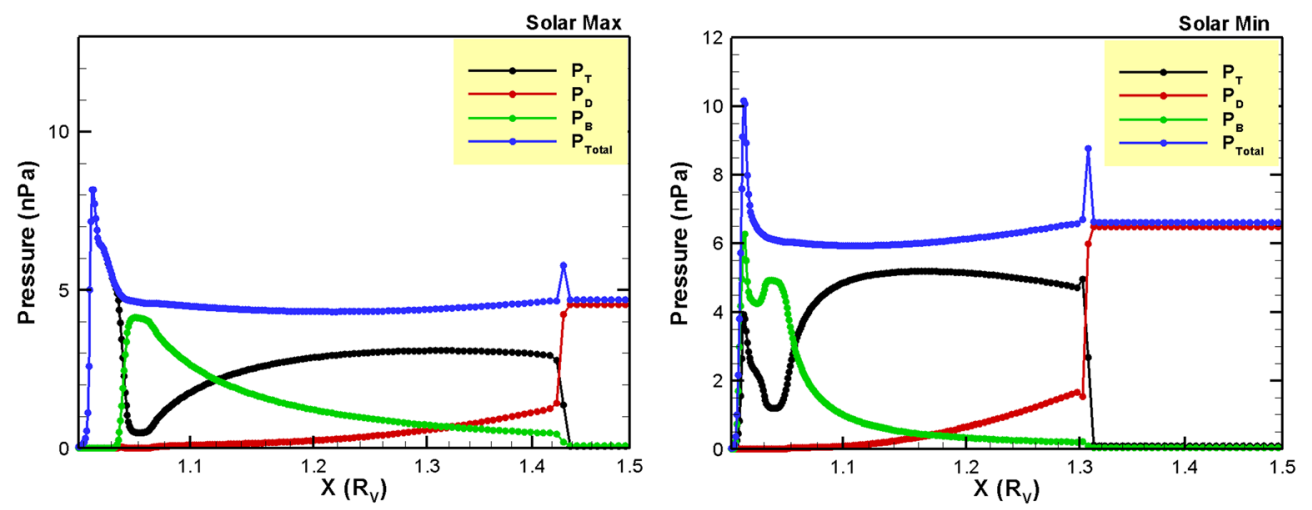

Figure 5. Pressure profiles as functions of distances along the subsolar line for solar max and solar min cases.

The corresponding collision rate, however, is estimated to be about three orders smaller than photo-ionization rates even for solar minimum case, so we may conclude that the effect of chemical processes is more critical. It is also important to note that the chemical processes are crucial to determine the density distribution of the ionosphere. We also ran our model with zero magnetic field in the solar wind for both cases to get results corresponding to a gas dynamic model. Simulation results show that the shock location is $1.33 R_{\mathrm{V}}$ and $1.27 R_{\mathrm{V}}$ for solar max and solar min conditions, respectively. This clearly demonstrates that the magnetic barrier that forms during the interaction acts as an additional obstacle that helps to stand off the solar wind even further, as speculated by Russell et al. [2006].

[25] Figure 5 shows the pressure profiles along the subsolar line for both cases. In the upstream solar wind, the plasma dynamic pressure $\left(P_{\mathrm{D}}=\rho U^{2}\right)$ plotted in red dominates plasma thermal pressure $\left(P_{\mathrm{T}}\right)$ in black and magnetic pressure $\left(P_{\mathrm{B}}=B^{2} / 2 \mu_{\mathbf{0}}\right)$ in green. Across the shock, the plasma kinetic energy is converted to plasma thermal energy and magnetic energy, as a result, the plasma thermal pressure and magnetic pressure increase while the plasma dynamic pressure drops sharply. The magnetic pressure keeps increasing inside the shock, while the plasma thermal pressure and dynamic pressure slowly decrease. Inside the ionopause, the ionospheric thermal pressure dominates. For solar max condition, the peak of the ionospheric thermal pressure is larger than the upstream solar wind dynamic pressure, while for the solar min case, it is the opposite. Consequently, the magnetic field behaves differently in the ionospheric region: in the solar max case, the magnetic field cannot penetrate the ionosphere, while in the other case, the magnetic field is significant inside the ionosphere. The peak magnetic field is $102 \mathrm{nT}$ and $125 \mathrm{nT}$ in the simulation during solar max and solar min respectively, and they are comparable to $91 \%$ and $97 \%$ of the corresponding solar wind dynamic pressure respectively.

[26] Figure 6 shows the calculated number density of ionospheric ions and protons along the subsolar line for both cases. The black circles indicate the radial grid points in the simulations. The ionosphere is mainly composed of $\mathrm{O}_{2}^{+}$below $200 \mathrm{~km}$ altitude. Above that altitude, $\mathrm{O}^{+}$becomes dominant. The peak density for solar max and solar min are $4.6 \times 10^{5} \mathrm{~cm}^{-3}$ and $2.6 \times 10^{5} \mathrm{~cm}^{-3}$, respectively. For the solar max case, the plasma density drops rather sharply at around $350 \mathrm{~km}$ as an indication of the ionopause, while for the solar min case, the sharp decrease happens near $250 \mathrm{~km}$. The location separating the ionosphere plasma from the ambient solar wind flow is usually called the ion composition boundary (ICB) [Martinecz et al., 2008]. The plasma outside of the ICB is dominated by protons, while mainly ionospheric ions
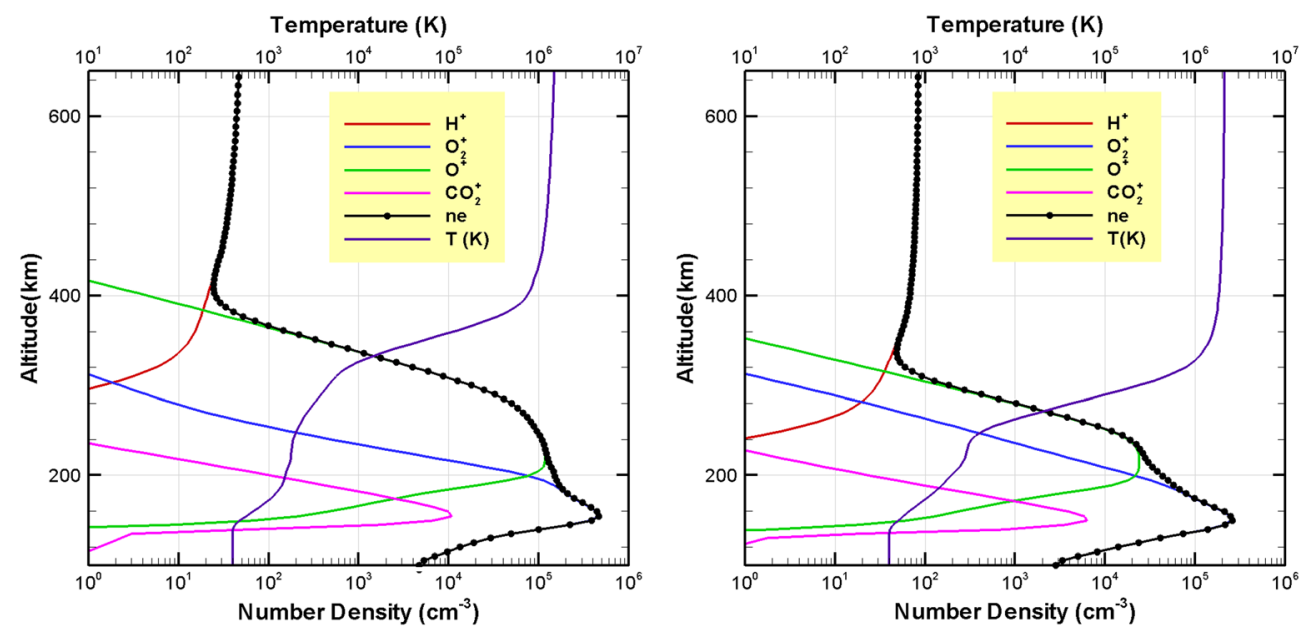

Figure 6. The calculated number density profiles as a function of altitude for the solar max and solar min cases along the subsolar line. 
(a)
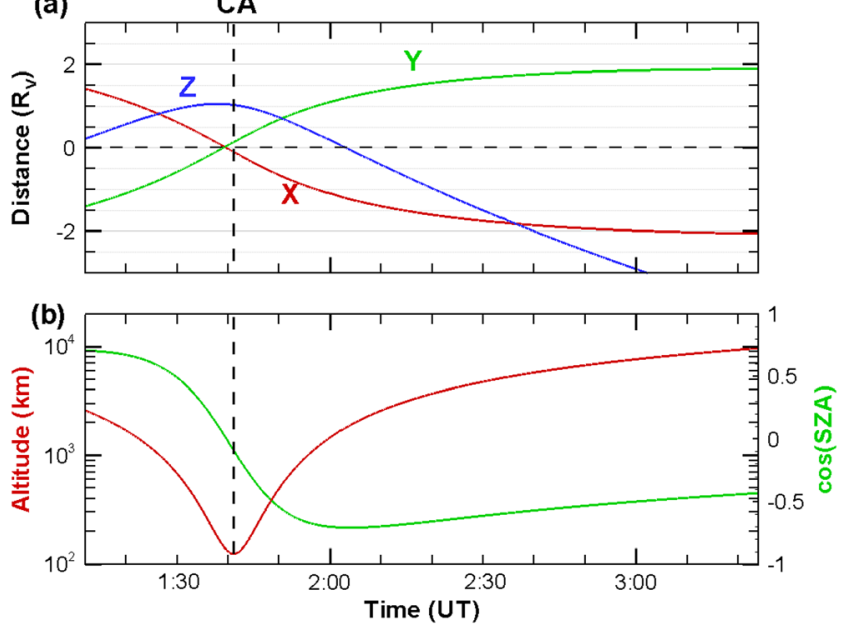

Figure 7. Orbit of VEX on 1 June 2006: (a) $X, Y, Z$ position of the spacecraft in Venus Sun Orbital coordinate; (b) altitude and $\cos (\mathrm{SZA})$ as functions of time.

are present inside the ICB. The ICB along the subsolar line is at $400 \mathrm{~km}$ for the solar max and $320 \mathrm{~km}$ for the solar min model.

\section{Comparison With VEX Results-A Case Study}

[27] We also compare our model results with VEX observations for a single orbit on 1 June 2006. A detailed description of the orbit can be found in Kallio et al. [2008]. The orbital parameters of the spacecraft are also shown in Figure 7. The vertical black dash line corresponding to 1:41 UT marks the time of the closest approach when the $\mathrm{S} / \mathrm{C}$ was $125 \mathrm{~km}$ above the surface near the north pole. We run our model using the set of upstream parameters based on the observations, as listed in Table 2 for case 3 . Such a set of parameters corresponds to a solar wind dynamic pressure of $3.7 \mathrm{nPa}$. The plasma beta is 2.6 and the fast magnetosonic Mach number is 6.3.

[28] The comparison of the model results with the magnetometer observations is shown in Figure 8 . The black lines show the measurement of $B_{X}, B_{Y}, B_{Z}$, and $B$ as a function of time. The blue lines are model results along the same trajectory. The model results show a sharp increase of $B_{\mathrm{X}}$ and $B$ across the inbound shock around 1:23 UT, which agree well with the observations. Both observed and modeled $B_{X}$ and $B$ gradually increase after the shock in the sheath region and piles up across the MPB (magnetic pile-up boundary). Around 1:55 UT, the observed $B_{X}$ suddenly changed from the positive to negative direction, indicating that the spacecraft crossed the current sheet from the north lobe to the south lobe. Both the reversal of the direction and the location of the current sheet are well reproduced by the model. Around 2:50 UT, the spacecraft crossed the outbound shock and went back into the solar wind. The $B_{X}$ component and magnetic field strength are well reproduced along the whole trajectory except between 1:37 UT and 1:41 UT near the pericenter, when the model predicts that the magnetic field $B_{X}$ component and $B$ drops to almost zero, while the observation show that magnetic field $B_{X}$ component and $B$ remain quite high. The discrepancy happens at an altitude below $200 \mathrm{~km}$ near the north polar region. This field could be an induced field, which diffuses into the lower altitude over a long period of time. Both the $B_{Y}$ and $B_{Z}$ components agree well with the observations. Overall, the location of the inbound shock, the peak of the magnetic field and the location of the current sheet and outbound shock are all reproduced by the MHD model.

[29] Figure 9 shows plasma parameters along the trajectory as predicted by the MHD model. The top panel shows the densities of protons $\left(\mathrm{H}^{+}\right)$in blue, oxygen $\left(\mathrm{O}^{+}\right)$in red and electrons $(\mathrm{Ne})$ in black as functions of time. Across the inbound shock, the proton density increases from $12 \mathrm{~cm}^{-3}$ to $39 \mathrm{~cm}^{-3}, 3.25$ times the solar wind density. The MHD model predicts that the $\mathrm{O}^{+}$density is dominant between 1:35 UT and 2:01 UT. Those two locations are the ICB (Ion composition boundaries) where the ions become dominated by planetary ions instead of solar wind protons. The electron number density first peaks at 1:37 UT with a value of $1.1 \times 10^{4} \mathrm{~cm}^{-3}$ where the $\mathrm{S} / \mathrm{C}$ is about $200 \mathrm{~km}$ altitude above the surface. At that time, the plasma is composed of more than $90 \%$ of $\mathrm{O}^{+}$ions and $10 \%$ of $\mathrm{O}_{2}^{+}$. The electron number density gradually decreases after the peak due to the increase of the SZA even though the spacecraft was getting closer to the planet. After periapsis, the electron number density drops more sharply because of the increase of the SZA and the spacecraft was moving away from the planet. The plasma density peaks again at 1:55 UT with a much

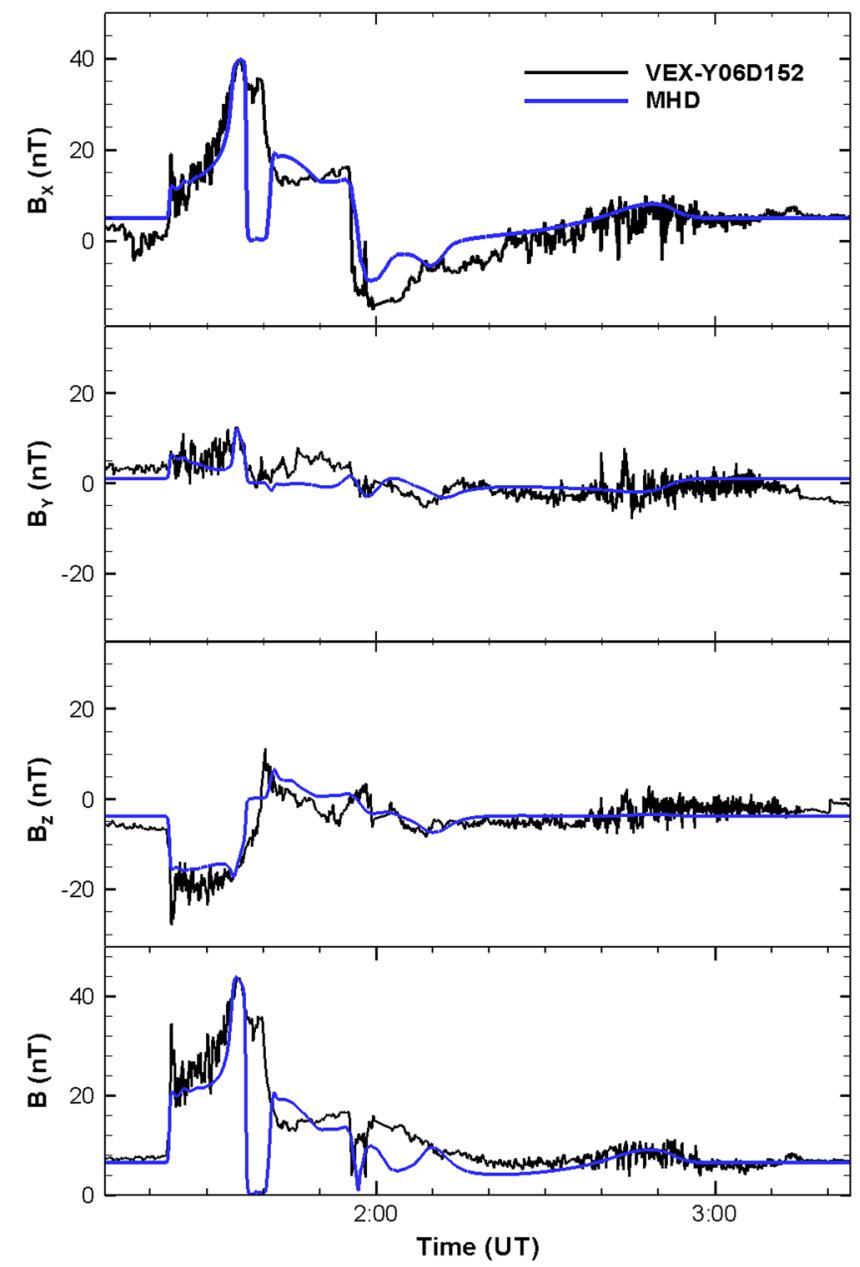

Figure 8. Comparison of MHD model results with the magnetometer observations along the trajectory. 

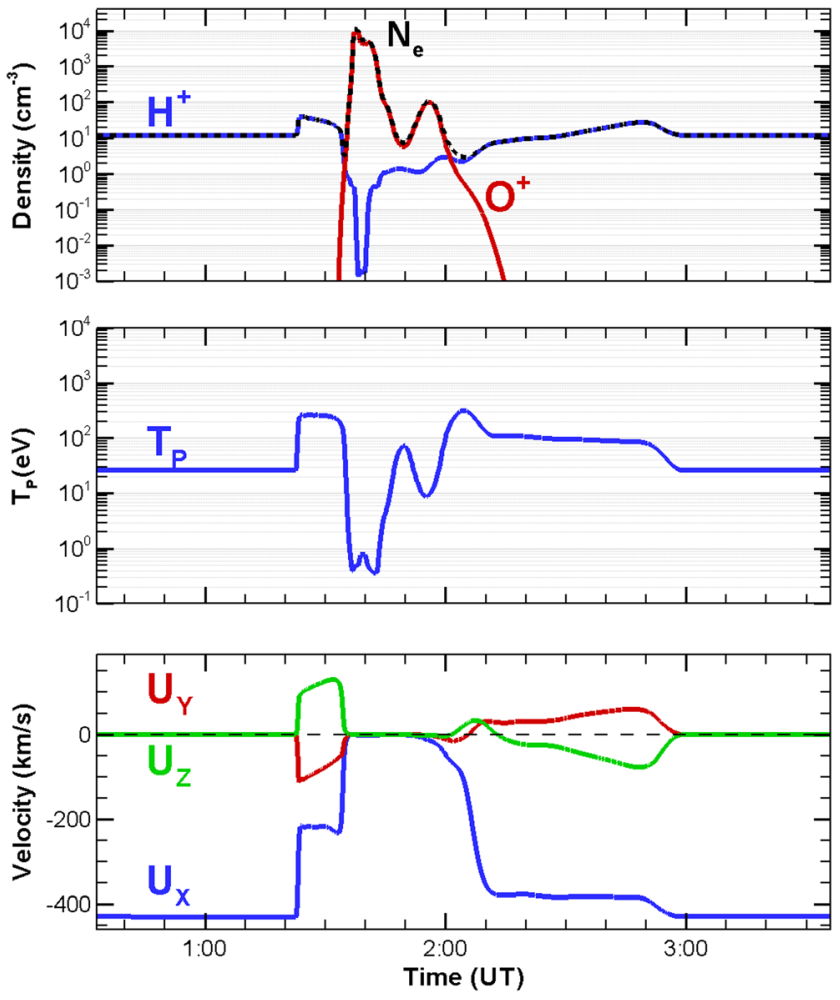

Figure 9. MHD model results of the plasma parameters along the trajectory.

smaller value of $10^{6} \mathrm{~cm}^{-3}$, in the center of the plasma current sheet in the wake region. The plasma density drops back to the solar wind value after the outbound shock.

[30] The middle and bottom panels are plasma temperature and velocity components along the trajectory. Across the shock, the plasma temperature increases, while the plasma flow speed drops to half of its original value and diverts around the obstacle. Across the ICB, the plasma temperature drops sharply because the plasma is mainly composed of cold planetary plasma. The plasma flow speed also drops to less than a few kilometers per second due to intense mass loading and ion-neutral inelastic collisions.

\section{Summary}

[31] We have developed a global multispecies MHD model to study solar wind interaction with the Venus ionosphere. The bow shock locations are well reproduced for both solar-maximum and solar-minimum conditions using appropriate solar wind parameters for each case. It is shown that the shock locations are farther from the planet during solar maximum conditions because of both the enhanced solar radiation strength and the relatively small Mach number. We also show that the both mass-loading and the magnetic barrier are important in producing the correct shock location.

[32] The simulation results also agree well with the Venus Express observations, as shown by comparisons between model results with magnetic fields observed by the spacecraft along a single orbit. The model results have also been used to understand the magnetometer observations along other orbits of VEX [Zhang et al., 2009]. The good agreement between model and data indicates that the interaction process can be reproduced quite well using simple neutral profiles for the Venus atmosphere. Some studies showed that kinetic effects [Kallio et al., 2006] could create asymmetric interaction patterns with regard to the convection electric field direction. Such effects are neglected in the single-fluid MHD model, but a multi-fluid MHD model with Hall effect included could also reproduce such an asymmetry [Jia et al., 2012]. Such effects will be included and tested in our future studies.

[33] Acknowledgments. The work was supported by NASA grants NNX10AB95G. Resources supporting this work were provided by the NASA High-End Computing (HEC) Program through the NASA Advanced Supercomputing (NAS) Division at Ames Research Center.

\section{References}

Bauske, R., et al. (1998), A three-dimensional MHD study of solar wind mass loading processes at Venus: Effects of photoionization, electron impact ionization and charge exchange, J. Geophys. Res., 103, 23,265.

Belotserkovskii, O. M., T. K. Breus, A.M. Krymskii, V. Y. Mitnitskii, A. F. Nagy, and T. I. Gombosi (1987), The effect of the hot oxygen corona on the interaction of the solar wind with Venus, Geophys. Res. Lett., 14, 503.

Breus, T. K., A. M. Krymskii, and V. Y. Mitnitskii (1987), Effect of an extended neutral atmosphere on the interaction of the solar wind and the nonmagnetic bodies of the solar wind, I, Venus, Kosm. Issled., 25, 124.

Cable, S., and R. S. Steinolfson (1995), Three-dimensional MHD simulations of the interaction between Venus and the solar wind, J. Geophys. Res., 100, 21,645.

Fox, J. L., and K. Y. Sung (2001), Solar activity variations of the Venus thermosphere/ionosphere, J. Geophys. Res., 106(A10), 21,305.

Jia, Y.-D., Y. J. Ma, C. T. Russell, H. R. Lai, G. Toth, and T. I. Gombosi (2012), Perpendicular flow deviation in a magnetized counter-streaming plasma, Icarus, 218(2), 895-905, doi:10.1016/j.icarus.2012.01.017.

Kallio, E., J. G. Luhmann, and J. G. Lyon (1998), Magnetic field near Venus: a comparison between PVO magnetic field observations and an MHD simulation, J. Geophys. Res., 103, 4723-4737.

Kallio, E., R. Jarvinen, and P. Janhunen (2006), Venus-solar wind interaction: asymmetries and the escape of $\mathrm{O}^{+}$ions, Planet. Space Sci., 54(13-14), $1472-148$.

Kallio, E., et al. (2008), The Venusian induced magnetosphere: A case study of plasma and magnetic field measurements on the Venus Express mission, Planet. Space Sci., 56(6), 796-801.

Luhmann, J. G., T.-L. Zhang, S. M. Petrinec, C. T. Russell, P. Gazis, and A. Barnes (1993), Solar cycle 21 effects on the Interplanetary Magnetic Field and related parameters at 0.7 and $1.0 \mathrm{AU}, J$. Geophys. Res., 98(A4), $5559-5572$.

Ma, Y., A. F. Nagy, I. V. Sokolov, and K. C. Hansen (2004a), Threedimensional, multispecies, high spatial resolution MHD studies of the solar wind interaction with Mars, J. Geophys. Res., 109, doi:10.1029/ 2002JA009293.

Ma, Y., A. F. Nagy, T. E. Cravens, I. V. Sokolov, J. Clark, and K. C. Hansen (2004b), 3-D global MHD model prediction for the first close flyby of Titan by Cassini, Geophys. Res. Lett., 31, L22803, doi:10.1029/ 2004GL021215.

Ma, Y., A. F. Nagy, T. Cravens, I. V. Sokolov, K. C. Hansen, J. Wahlund, F. Crary, A. Coates, and M. Dougherty (2006), Comparisons between MHD model calculations and observations of Cassini flybys of Titan, J. Geophys. Res., 111(A5), A05207.

Ma, Y., and A. F. Nagy (2007), Ion escape fluxes from Mars, Geophys. Res. Lett., 34, L08201, doi:10.1029/2006GL029208.

Ma, Y., et al. (2007), 3D global multi-species Hall-MHD simulation of the Cassini T9 flyby, Geophys. Res. Lett., 34, L24S10, doi:10.1029/ 2007GL031627.

Martinecz, C., et al. (2008), Location of the bow shock and ion composition boundaries at Venus - initial determinations from Venus Express ASPERA-4, Planet. Space Sci., 56(6), 780-784.

McGary, J. E., and D. H. Pontius Jr. (1994), MHD simulations of the boundary layer formation along the dayside Venus ionopause due to mass loading, J. Geophys. Res., 992289

Murawski, K., and R. S. Steinolfson (1996), Numerical simulations of mass loading in the solar wind interaction with Venus, J. Geophys. Res., 101, 2547.

Phillips, J. L., and C. T. Russell (1987), Revised upper limit on the internal magnetic moment of Venus, Adv. in Space Res., 7(12), 291-294.

Powell, K. G., P. L. Roe, T. J. Linde, T. I. Gombosi, and D. L. DeZeeuw (1999), A solution- adaptive upwind scheme for ideal magnetohydrodynamics, J. Comp. Phys., 154, 284. 


\section{MA ET AL.: MHD STUDY OF PLASMA INTERACTION AROUND VENUS}

Russell, C. T., J. G. Luhmann, and R. J. Strangeway (2006), The solar wind interaction with Venus through the eyes of the Pioneer Venus Orbiter, Planet. Space Sci., 54, 1482-1495.

Schunk, R. W., and A. F. Nagy (1980), Ionospheres of the terrestrial planets, Rev. Geophys., 18(4), 813-852, doi:10.1029/RG018i004p00813.

Schunk, R. W., and A. F. Nagy (2009), Ionospheres, 2nd edn, Cambridge University Press, Cambridge.

Slavin, J. A., et al. (1980), The solar wind interaction with Venus-Pioneer Venus observations of bow shock location and structure, J. Geophys. Res., 85, 7625-7641.

Spreiter, J. R., and S. S. Stahara (1980), Solar wind flow past Venus: Theory and comparisons, J. Geophys. Res., 85, 7715.

Spreiter, J. R., and S. S. Stahara (1992), Computer modeling of solar wind interaction with Venus and Mars, in Venus and Mars: Athmospheres, Ionospheres and Solar Wind Interactions, Geophys. Monogr. Set, edited by J. G. Luhmann, M. Tatrallyay, and R. O. Pepin, vol. 66, p. 345, AGU, Washington D.C.

Stahara, S. S., G. A. Molvik, and J. R. Spreiter (1987), A new computational model for the prediction of mass loading phenomena for the solar wind interactions with cometary and planetary ionospheres, AIAA 87-1410, Am. Inst. of Aeronaut. and Astronaut., New York.

Strangeway, R. J., and C. T. Russell (1996), Plasma waves and field-aligned currents in the Venus plasma mantle, J. Geophys. Res., 101(A8), 17,313-17,324, doi:10.1029/96JA00927.

Tanaka, T., and K. Murawski (1997), Three-dimensional MHD simulation of the solar wind interaction with the ionosphere of Venus: Results of two-component reacting plasma simulation, J. Geophys. Res., 102, 19,805.
Tanaka, T. (2000), Effects of Decreasing Ionospheric Pressure and the Plasma Mixing Recess on the Solar Wind Interaction with Non-magnetized Planets, Advances in Space Res., 26(10), 1577-1586.

Terada, N., S. Machida, and H. Shinagawa (2002), Global hybrid simulation of the Kelvin-Helmholtz instability at the Venus ionopause. J. Geophys. Res., 107(A12), 1471.

Terada, N., H. Shinagawa, and S. Machida (2004), Global hybrid model of the solar wind interaction with the Venus ionosphere: ion escape processes, Adv. Space Res., 33(2), 161-166.

Terada, N., H. Shinagawa, T. Tanaka, K. Murawski, and K. Terada (2009), A three-dimensional, multispecies, comprehensive MHD model of the solar wind interaction with the planet Venus, J. Geophys. Res., 114, A09208, doi:10.1029/2008JA013937.

Tóth, G., et al. (2012), Adaptive Numerical Algorithms in Space Weather Modeling, J. Comput. Phys., 231, 870-903.

Zhang, T.-L., J. G. Luhmann, and C. T. Russell (1990), The solar cycle dependence of the location and shape of the Venus bow shock, J. Geophys. Res., 95, 14,961-14,967.

Zhang, T.-L., J. G. Luhmann, and C. T. Russell (1991), The magnetic barrier at Venus, J. Geophys. Res., 96, 11,145-11,153.

Zhang, T.-L., et al. (2008), Initial Venus Express magnetic field observations of the Venus bow shock location at solar minimum, Planet. Space Sci., 56(6), 785-789.

Zhang, T. L., J. Du, Y. J. Ma, H. Lammer, W. Baumjohann, C. Wang, and C. T. Russell (2009), Disappearing induced magnetosphere at Venus: Implications for close-in exoplanets, Geophys. Res. Lett., 36, L20203, doi:10.1029/2009GL040515. 